their necks from one another by the deep epigastric vessels (Fig. 2).

The operations performed on the inguinal herniæ were various. In 79 cases I employed my own method, previously described; in 57, Bassini's method following very closely his original description in Langenbeck's Archiv, except in two small details, in which I venture to think I have introduced a slight improvement. In 7 cases Kocher's operation was done following his own modifications as he published them. In only 2 has Dr. Macewen's method been followed. The remaining 10 cases were operated on in the earlier days by irregular methods of suture of the rings before the operations became formulated.

In the femoral, umbilical, and ventral cases I have followed methods I described some years ago, and have only modified them so far as in the last 9 cases of ventral and umbilical cases to use deep buried silver sutures, following a practice from which $I$ have seen very good results in the hands of Professor Max Schede, formerly of Hamburg, now at Bonn. The silver wires appear to me as to him to give a decided mechanical support not obtained by silk. In one case in which I had to operate for incarcerated umbilical hernia in a stout woman five months pregnant, I was greatly struck with the result. She went through her labour without any trouble in the hernia, and I saw her ten months later, with her 5 months' old baby, and could find no trace of the hernia or silver stitches. In one stout man who unfortunately was operated on for umbilical hernia a few hours after admission into hospital, and whose urine had not, by some mistake, been examined, it was found after operation that he had diabetes. Here the wound slowly suppurated, and the silver stitches had to be removed. This case, and one more of a neurotic woman, who thought that the silver stitches pinched her, are the only cases in which they have had to be removed. And to me they appear ${ }_{c} \mathrm{to}_{a}^{\prime 3}$ have $e_{\alpha}$ done good service. A larger experience will tell.

In almost every other case hard twist Chinese silk boiled in I to 20 absolute phenol was used, my earlier experiences with gut of all kinds not impressing me favourably. Some of these silk sutures, as has been said above, worked out to the surface ; but, considering that about a thousand must have been used in the 200 operations the proportion was small.

(3) As to the last question put in regard to the immunity from recurrence after these operations, it is an extremely difficult one to answer. Most of these cases, of course, were hospital patients, and to follow them up has been next to impossible. But this much I can say, namely, that I constantly hear of my patients from various parts of the world in every profession and employment who are quite rid of all their troubles, and that I very rarely now hear of a recurrence. Whether this is due to the fact that Bassini's operation has been the method I cannot say, but I certainly regard it, when carefully carried out, as the best operation of any yet devised.

\section{A CASE OF NOMA OF THE EAR.}

By G. MUNRO SMITH, L.R.C.P.LoND., M.R.C.S., Surgeon to the Bristol Royal Infirmary.

The unusual situation of the lesion in this case and the result of the bacteriological examination make it worthy of record.

\section{History.} M. A. V., aged 2 years, a fairly well nourished but rather anæmic child,
was admitted to the Bristol Royal Infirmary, September 15 th, 1897 . There was admitted to the Bristol Royal Infirmary, September 15 th, 8897 . There was a history of occasional discharge from the left ear and eczema, otherwise she had been healthy. The mother had suffered from syphilis, and others of her children had been ill with enlarged gland

etc. No account of any local injury could be obtained. On admission, the whole of the left auricle was swollen and red, and pus was exuding from the meatus; the cheek was œedematous. There was a small, clean-cut ulcer in the fossa of the antihelix, covered with a greenish grey, slightly adherent slo

The ulcer was cleaned, antiseptic dressings were applied, and matters The ulcer was cleaned, antiseptic dressings were applied, and matters improved until September 25 th, when the auricle began rapidly to swell and the patient became worse. On the $26 \mathrm{th}$, an incision was made and a teaspoonful of pus evacuated. On October ist the ulcer began to was scraped and cut away and strong carbolic applied. There was another improvement for a few days, but on October 4 th the disease made a fresh start, and from this time onwards its ravages could.only be temporarily checked by cutting away the necrosed tissues and swabbing with strong antiseptics. The condyle of the jaw and neighbouring parts became ex- posed, and on October ${ }_{27}$ th symptoms of meningitis appeared, and, the child died on the 29th.

At the necropsy the external ear was represented by a small," "crescenthaped mass, and a large irregular cavity, with sharply excavated sides. the of the temporal bone (see Fig. I). The articulation was destroyed, but the

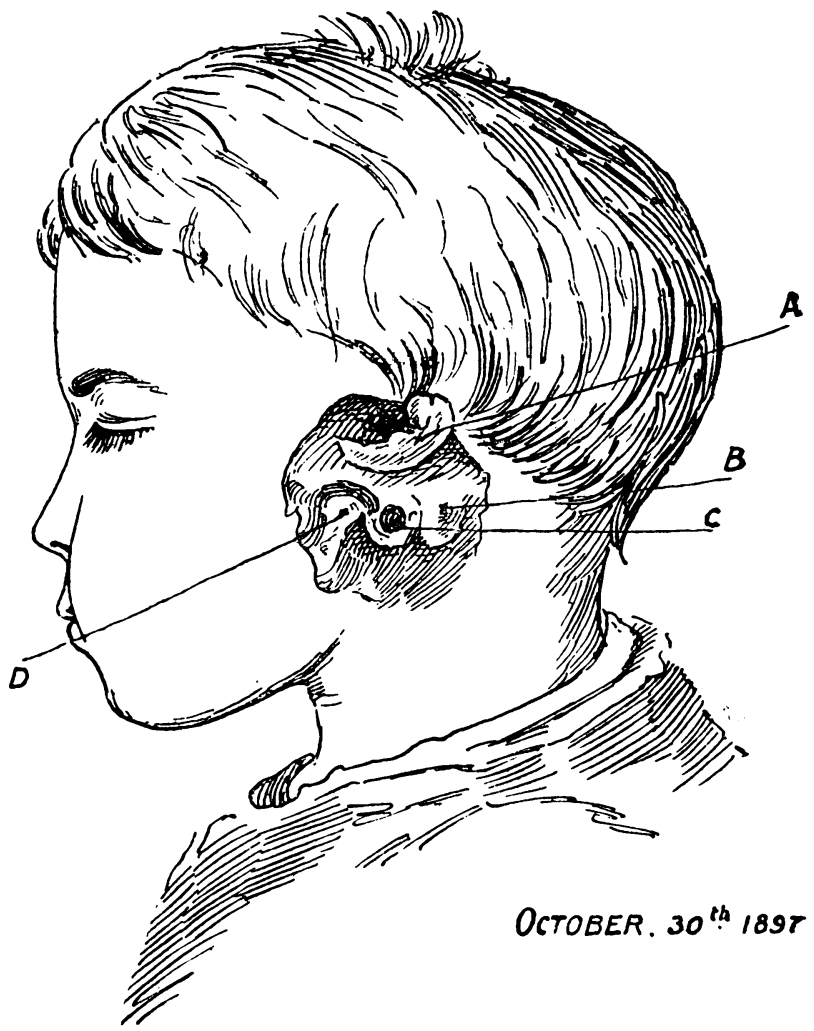

Fig. I. $-\Lambda$, Remains of auricle. B, Mastoid. C, External auditory meatus. D, Condyle of lower jaw.

tympanum had escaped. On opening the cranial cavity the vessels of the vertex of the brain were much congested, and the lateral sinus was blocked with white adherent clot. There was also a thrombus in the longitudinal sinus.

\section{REMARKS.}

The clinical features were fairly typical of noma, but there was an almost complete absence of the black slough generally found and the situation was very unusual. I can find no record of the external ear being primarily attacked. The extensive thrombosis must be considered one of the features of the disease.

Various bacteriologists have found micro-organisms in the tissues involved in noma and in the clots in neighbouring veins, but their relation to the affection is not yet clearly defined. However, in 1889 Schimmelbusch described small bacilli with rounded ends which stained with aniline dyes, but not with Gram's method. Injections into mice and pigeons caused sloughing, etc. ${ }^{1}$ Ranke in several cases found only cocci. ${ }^{2}$

Dr. Odery Symes, Honorary Bacteriologist to the Bristol Royal Infirmary, kindly undertook the bacteriological examination, and I am indebted to him for the following report and to the House Physician, Dr. Stack, for taking notes on the daily progress of the disease.

Bacteriological Report.

Dr. Stack inoculated an agar tube from the margin of the spreading ulcer on October 27th. After twenty-four hours incubation at $37^{\circ} \mathrm{C}$., there was a growth of greyish-white colonies. Examined microscopically, there were a few staphylococci present, together with a short bacillus, nonmotile, rounded at the ends, and staining easily with aniline dyes. The centre of the bacillus often remains unstained. The organisms are often in pairs or chains. On gelatine stroke cultures the growth is very slow, and does not liquefy; 
the colonies are greyish-white, granular, and glistening. In gelatine stab cultures the colonies are white. At the postmortem examination, nineteen hours after death, cultures were taken from the spleen and from the thrombus in the longitudinal sinus. The agar tube inoculated from the spleen, after a week's incubation, was still sterile. The culture from the longitudinal sinus gave a pure culture of the bacillus described above. This organism resembles in all points that described by Schimmelbusch as associated with cancrum oris. That described by Lingard is, perhaps, the same.

Dr. Symes forwarded a tube to Mr. Plimmer, hoping that some inoculation experiments might be made, but unfortunately the tube was broken and contaminated in transit, and cultures failed; but the organism was identified by $\mathrm{Mr}$. Plimmer as that described by Schimmelbusch. Fig. 2 is a

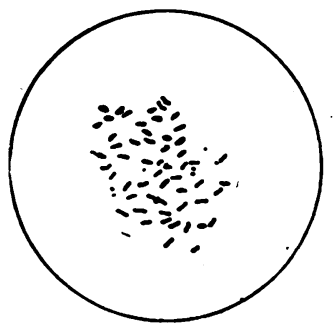

Fig. 2.-Cultivation on agar. (Hartnack : Leitz's oil immersion, $\frac{1}{2}$.) drawing I made of these bacilli.

REFERENCES

1 Schimmelbusch, Deutsch med. Woch., r889. 2 Ranke, Jahrb. f. Kinderheilk., 1888 .

\section{HEREDITARY DIGITAL ABNORMALITY.}

BY D. YOUNG, M.D., Parkhead, Glasgow.

A CASE of abnormality of the thumb having come under my notice some time ago, I instituted inquiries as to the occurrence of similar conditions in near relatives, with the result that the following remarkable series of cases was elicited. The hereditary tendency is abundantly demonstrated, and it is rather interesting to note that the peculiarities have been transmitted almost invariably from the paternal side.

First Generation.-The earliest instance which can be recalled in this family is in the paternal great-grandfather. He had what the family calls "straight thumbs;" this term may be retained, and I offer a few words in its explanation, as three of the descendants presenting it have come under my own observation. The terminal joint is ankylosed, and both phalanges are broad, as if two thumbs had been begun to be formed, producing, however, single flat phalanges with a single broad nail ; the first phalanx, moreover, is longer than normal, while the terminal phalanx is short.

Second Generation.-His sister's son (nephew) has grown up with two distinct thumbs on each hand, so that probably the condition goes back to five generations. His daughter has the right thumb bent in like a claw, and his son has "straight thumbs."

Third Generation.- The son last mentioned has a son with " straight thumbs;" the daughter has five children, three of whom are affected ; two sons have "straight thumbs," and the daughter an enlarged right thumb turned in (clawed). In the two other sons I know of no defect.

Fourth Generation.-Of these two sons, who themselves did not present the abnormality, one had a boy with doublewebbed thumbs on each hand, upon whom I operated successfully, and the other a boy with webbed fingers. One of the sons who had "straight thumbs" has a family of eight, three of whom are affected. The first child (boy) had two distinct thumbs on each hand, and was operated upon by Dr. Turnbull, of Cambuslang, the remaining thumbs being "straight." He is a violinist, and in tuning his instrument catches the keys between the metacarpal bone and hand; in addition, he has on each foot a double-webbed great toe with two nails. The sixth child (girl) had two distinct thumbs on each hand, separate metacarpal joint for each, on whom I operated successfully, and she also has double-webbed great toes. The eighth child (boy 3 weeks old) has "straight thumbs," the terminal joint, however, being a little mobile. I have advised the parents to exercise it; he has also doublewebbed great toes. Thus one man with "straight thumbs" transmits through the next three generations digital peculiarities to at least eleven descendants. It would be interesting to know the results which might follow intermarriage of any of these.

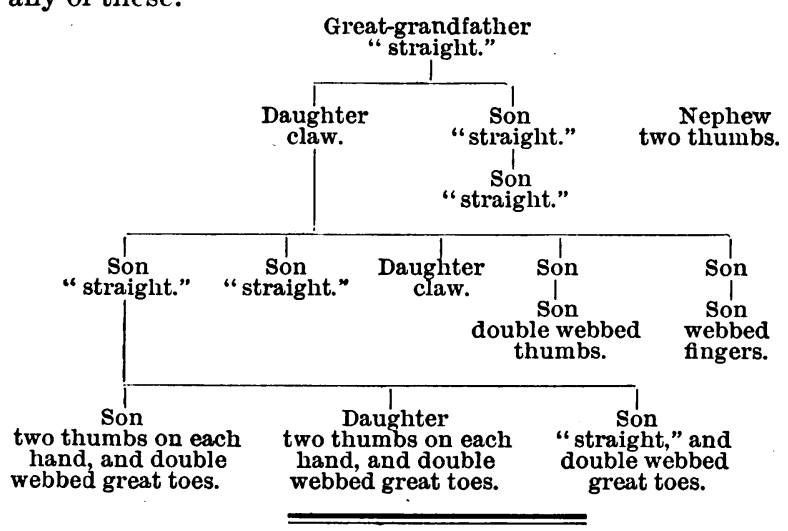

\section{MEDICATED WINES.}

BY FREDERIC C. COLEY, M.D.,

Physician to the Hospital for Sick Children, Newcastle-upon-Tyne, and to the Northern Counties Hospital for Diseases of the Chest.

I HAVE been very glad to have seen more than once of late in the British MEDIOAL JooRNAL protests against the general use of certain fluids largely advertised under the name of medicated wines. There are two classes of such preparations which I think it is not only our duty to avoid recommending, but against which we should warn our readers with the greatest earnestness, and be prepared to explain our reasons for so doing.

The first class to which I allude are called coca wines, compounds which consist of a wine of considerable alcoholic strength (generally, I believe, claiming to be either port or sherry), with the soluble parts of coca, or else a salt of cocaine dissolved therein. Coca and its chief alkaloid, cocaine, are drugs which possess some power of removing the sense of fatigue, just as analgesics remove the consciousness of pain. But they no more remove the physical condition of muscles and nerve centres of which the sense of pain gives us warning than a dose of morphine which relieves the pain of toothache removes the offending tooth, or even arrests the caries in it. The truth of this will be obvious to anyone who remembers enough of physiology to know what fatigue really means. A muscle which is tired out is different chemically from the same muscle in its more normal condition, when it is ready to respond vigorously to ordinary stimuli. It has lost something and is besides overcharged (poisoned, in fact) with the products of its own activity, and it can only be restored by a fresh supply of the material which it requires, and the carrying away of the poisonous waste products. Fatigue of nerve centres is no doubt strictly analogous to fatigue of muscles.

It is practically impossible for us by voluntary exertion to reach the degree of absolute fatigue which the physiologist produces by electric stimulation of a nerve-muscle preparation. The sense of fatigue becomes so intense that voluntary effort cannot overcome it, just as no man can produce asphyxia by simply holding his breath, because the besoin de respirer becomes irresistible; but it is quite possible for a narcotic to so dull the sensory part of the respiratory reflex mechanism as to permit asphyxia to take place.

The sense of fatigue and the besoin de respirer are both Nature's danger signals. Drugs which hide such signals from us are a more than doubtful benefit. If it were possible for us to suppose that a fraction of a grain of cocaine could afford to exhausted nerve centres and muscles the nutriment which they require for their restoration, and at the same time eliminate the poisonous waste products, then it would be reasonable to prescribe the drug for use by all who are over- 